\title{
Entwicklung eines kostengünstigen Gaschromatographie-Systems für die Messung von Reifegas mit Kompensation der Temperatureinflüsse auf die Säule.
}

\author{
Maximilian Köhne ${ }^{1}$, Gina Zeh ${ }^{1}$, Christopher Schmidt ${ }^{1}$ und Tilman Sauerwald ${ }^{1,2}$ \\ ${ }^{1}$ Fraunhofer Institut für Verfahrenstechnik und Verpackung, Freising, Deutschland \\ ${ }^{2}$ Universität des Saarlandes, Saarbrücken, Deutschland \\ Kontakt: maximilian.koehne@ivv.fraunhofer.de
}

\section{Einleitung}

Ethen (auch Ethylen genannt) besitzt als Phytohormon eine hohe Bedeutung für den Reifungsprozess klimakterischer Früchte. Es fördert die Fruchtreife, kann aber auch die Seneszenz einleiten und somit zum Verderb der Früchte beitragen [1, 2]. Dabei wirkt das Ethen selbstverstärkend, da es zum einen die Reife induziert und zum anderen als Stoffwechselprodukt mit der fortschreitenden Reife gebildet wird [3]. Neben seiner Eigenschaft als Hormon kann es daher auch als Marker für den Reifungsgrad der Früchte und somit für deren Qualität herangezogen werden. Eine Überwachung der Ethenbildung nach der Ernte kann somit die Fruchtqualität sicherstellen und die Entwicklung der Fruchtqualität prognostizieren [4, 5].

Deswegen spielt die Messung von Ethen als Reifungsgas klimakterischer Früchte, wie Äpfel, Avocados, Tomaten, Bananen, etc. im fefo-Prozess (first expired first out, engl. für „zuerst abgelaufen zuerst raus“) in der Lebensmittellogistik eine zentrale Rolle zur Vermeidung unnötiger Abfälle [6, 7]. Durch z.B. weite Transportwege tropischer Früchte von Südamerika nach Europa und dem aktuell gängigen first in first out (engl. für „zuerst rein, zuerst raus“) Prinzip, entstehen jährlich größere Mengen an Abfall durch den frühzeitigen Verderb einiger Chargen. Dabei können durch den fefo-Prozess im Reifungsstadium weiter fortgeschrittene Obstchargen mithilfe der Ethendetektion früher in den Handel gelangen als unreifere Chargen. Die Wahrscheinlichkeit des Verderbs ganzer Chargen wird so durch eine produktspezifischere Zustandserkennung verringert, was die entstehende Abfallmenge reduzieren kann [5, 8, 9].

Kleine, analytische Geräte, wie kostengünstige Gaschromatographen (GCs) können daher zur Zustandserkennung der Reife klimakterischer Früchte durch die Ethendetektion beitragen [9-12]. Gaschromatographen sind dabei nötig, um eine ausreichend gute Unterscheidung des Ethens von anderen Gasen und Störfaktoren wie Feuchtigkeit zu erzielen [9]. Um aber solche kleinen GCs in einem größeren Umfang einsetzen zu können, müssen diese zum einen miniaturisiert und robust gestaltet werden. Zum anderen ist ein geringer Bauteilaufwand für die Kostenersparnis der GCs erforderlich. Eine Möglichkeit zur Kostenreduktion besteht dabei in einer Kompensation der Temperatureinflüsse auf die Säule der GC-Einheit. Eine normalerweise nötige präzise Temperaturregelung der Säule wird überflüssig, wodurch Bauteile und Materialkosten eingespart werden können. Im Rahmen der Entwicklung eines miniaturisieren GCs für die Ethenmessung mit einer PLOT-Säule (engl. porous layer open tubular; poröse Schicht, offenes Rohr) und zwei IR-Detektoren, wurde daher die Kompensation der Temperatureinflüsse durch die Retentionsindizes von Test- und Referenzgasen auf einer GC-Säule modelliert und anschließend an einem klassischen GC mit einem Pulsed Discharge Detektor (PDD) getestet. Als Referenzgas wurde dabei natürlich vorkommendes $\mathrm{CO}_{2}$ gewählt, da es ubiquitär und leicht detektierbar ist.

\section{Temperaturkompensationsmodell}

Die aus den Messdaten berechnete Abhängigkeit der Gase voneinander ermöglicht die Bestimmung der EthenRetentionszeit (RTEthen) anhand der $\mathrm{CO}_{2}$-Retentionszeit $\left(\mathrm{RT}_{\mathrm{CO}_{2}}\right)$ und damit die Berechnung eines von der Säulentemperatur unabhängigen Parameters. Die Berechnung erfolgt über eine van't Hoff Gleichung. Dabei werden die Entropie $S$ und die Enthalpie $H$, die den Sorptionsprozess beschreiben, mit Hilfe des temperaturabhängigen Gleichgewichtskoeffizienten $\mathrm{K}$ berechnet (1).

$$
\Delta H^{0}-T \Delta S^{0}=-R T * \ln (K(T))
$$

Praktisch lassen sich diese Parameter als Koeffizienten der Geradengleichung von $\ln \left(K\left(\frac{1}{T}\right)\right)$ bestimmen (2):

$$
\ln \left(K\left(\frac{1}{T}\right)\right)=-\frac{\Delta H^{0}}{R} * \frac{1}{T}+\frac{\Delta S^{0}}{R}
$$

Der Gleichgewichtskoeffizient K kann über einfache Testmessungen am GC bestimmt werden und ist proportional zum Nettoretentionsvolumen $V_{r}^{\prime}$ und bei konstantem Fluss $\vartheta$ zur Nettoretentionszeit $t_{r}^{\prime}(3)$.

$$
K=\frac{V_{r}^{\prime}}{V_{S}}=\frac{\vartheta * t^{\prime} r}{V_{S}}=\frac{\vartheta *\left(t_{r}-t_{0}\right)}{V_{S}}
$$

Die Nettoretentionszeit $t_{r}{ }^{r}$ ist die Differenz aus der (gemessenen) Bruttoretentionszeit $t_{r}$, und der Totzeit to. Die Totzeit lässt sich guter Näherung aus der Retentionszeit von Stickstoff bestimmen, da dieser mit der hier verwendeten PLOTSäule kaum interagiert. Durch die Testmessungen mit dem entsprechenden Referenzgas und dem Testgas lassen sich 
also die entsprechenden Adsorbtionsenthalpien und die Entropien der jeweiligen Gase auf der gewählten Säule bestimmen. Durch Auflösung der Gleichung (2) nach $\frac{1}{T}$ können die entsprechenden Terme für $\mathrm{CO}_{2}$ und Ethen gleichgesetzt werden. Man erhält so Gleichung (4):

$$
-\frac{\ln \left(K_{E}\right) R}{\Delta H_{E}^{0}}+\frac{\Delta S_{E}^{0}}{\Delta H_{E}^{0}}=-\frac{\ln \left(K_{C}\right) R}{\Delta H_{C}^{0}}+\frac{\Delta S_{C}^{0}}{\Delta H_{C}^{0}}
$$

Wobei der Index E für Ethen und der Index $\mathrm{C}$ für $\mathrm{CO}_{2}$ steht. Durch das Auflösen von (4) nach $\mathrm{K}_{\mathrm{E}}$ erhält man:

$$
K_{E}=\exp \left(\left[\frac{\ln \left(K_{C}\right) R-\Delta S_{C}^{0}}{\Delta H_{C}^{0}}\right] * \frac{\Delta H_{E}^{0}}{R}+\frac{\Delta S_{E}^{0}}{R}\right)
$$

Mithilfe der Gleichungen (3) und (5) lässt sich nun die Retentionszeit des Ethens in Abhängigkeit der Retentionszeit des $\mathrm{CO}_{2}$ prognostizieren:

$$
t_{r E}^{\prime}=\frac{V_{S}}{\vartheta} * \exp \left(\left[\frac{\ln \left(\frac{t_{r C}^{\prime} * \vartheta}{V_{S}}\right) * R-\Delta S_{C}^{0}}{\Delta H_{C}^{0}}\right] * \frac{\Delta H_{E}^{0}}{R}+\frac{\Delta S_{E}^{0}}{R}\right)(6)
$$

So ist es nun möglich mithilfe der Retentionszeit des $\mathrm{CO}_{2}$ die Retentionszeit des Ethens vorherzusagen.

\section{Methoden und Materialien}

Zum Test des beschriebenen Kompensationsalgorithmus und zur Ermittlung der notwendigen Parameter wurden Messungen mit $\mathrm{CO}_{2}$ und Ethen als Referenz- und Testgas durchgeführt. Dazu wurden $100 \mu \mathrm{L} / \mathrm{L}$ Ethen (Testgas) mittels einer gasdichten Spritze (Hamilton, Gastight, $100 \mu \mathrm{L}$ ) in eine Gasmesszelle (4 L Volumen) injiziert (siehe Abb. 1). Als Referenzgas wurde in der Umgebungsluft vorkommendes $\mathrm{CO}_{2}$ (ca. 400 ppm) gewählt, da es zusammen mit der gesamten Gasprobe aus der Gasmesszelle über die Gasdosierschleife der GC-Einheit entnommen werden konnte. Außerdem kann $\mathrm{CO}_{2}$ so auch für miniaturisierte GC-Systeme zugänglich gemacht werden. Die Entnahmemenge pro Messung betrug $5 \mathrm{~mL}$. Zur gleichmäßigen Gasdurchmischung wurde ein Magnetrührer mit einem Segel aus Aluminiumfolie $(5 \times 5 \mathrm{~cm})$ ausgestattet und die Probe vor jeder Messung bei $1200 \mathrm{U} / \mathrm{min}$ für 7 min gerührt. Die temperaturabhängigen Retentionszeiten des Testgases Ethen und des Referenzgases $\mathrm{CO}_{2}$ wurden anschließend in einem GC (Fisons Instruments, GC 8000 series) mit einer GS-Q Säule (GS-Q Megabore, 0,53 mm ID, J\&W Scientific) und einem PDD (VICI Valco Instruments Co. Inc.) auf einem HeliumTrägergasstrom gemessen. Der Detektor gibt dabei ein Wandlersignal im $\mathrm{mV}$ Bereich aus. Zur Erstellung des van't Hoff Diagramms wurden je drei Messungen bei $32^{\circ} \mathrm{C}$ (betrieblich bedingtes Minimum des GC-Ofens), $35^{\circ} \mathrm{C}$ und in $5{ }^{\circ} \mathrm{C}$ Schritten bis $50{ }^{\circ} \mathrm{C}$ gemessen. Zur Überprüfung des van't Hoff Diagramms wurden außerdem die Temperaturen $37^{\circ} \mathrm{C}, 42^{\circ} \mathrm{C}$ (Interpolation) und $60^{\circ} \mathrm{C}$, sowie $70{ }^{\circ} \mathrm{C}$ (Extrapolation) gemessen.
Tab. 1: Zusammensetzung Störgasmischung

\begin{tabular}{|l|c|l|}
\hline \multicolumn{2}{|c|}{ Gas-Komponente } & Menge \\
\hline $\mathrm{O}_{2}$ & (Sauerstoff) & 5000 Vol. ppm \\
\hline $\mathrm{C}_{2} \mathrm{H}_{2}$ & Ethin (Acetylen) & 5000 Vol. ppm \\
\hline $\mathrm{CO}$ & Kohlenstoffmonoxid & 5 Vol. \% \\
\hline $\mathrm{CH}_{4}$ & Methan & 5 Vol. $\%$ \\
\hline $\mathrm{CO}_{2}$ & Kohlenstoffdioxid & 5 Vol. \% \\
\hline $\mathrm{C}_{2} \mathrm{H}_{4}$ & Ethen & 5 Vol. \% \\
\hline $\mathrm{C}_{2} \mathrm{H}_{6}$ & Ethan & 5 Vol. \% \\
\hline $\mathrm{N}_{2}$ & Stickstoff & 5 Vol. $\%$ \\
\hline $\mathrm{He}$ & Helium & Rest \\
\hline
\end{tabular}

Für die Messungen mit Störgasen wurden zusätzlich zu den $100 \mu \mathrm{L} / \mathrm{L}$ Ethen $16 \mathrm{ml}$ aus einer Gasmischung (Westfalen AG, Zusammensetzung siehe Tab. 1) injiziert. Die Messungen erfolgten bei $32{ }^{\circ} \mathrm{C}, 35^{\circ} \mathrm{C}$ und anschließend in $5{ }^{\circ} \mathrm{C}$ Schritten bis $70^{\circ} \mathrm{C}$. Die Identifikation des Ethens erfolgte mittels der in der ersten Messung bestimmten van't Hoff Diagramme.
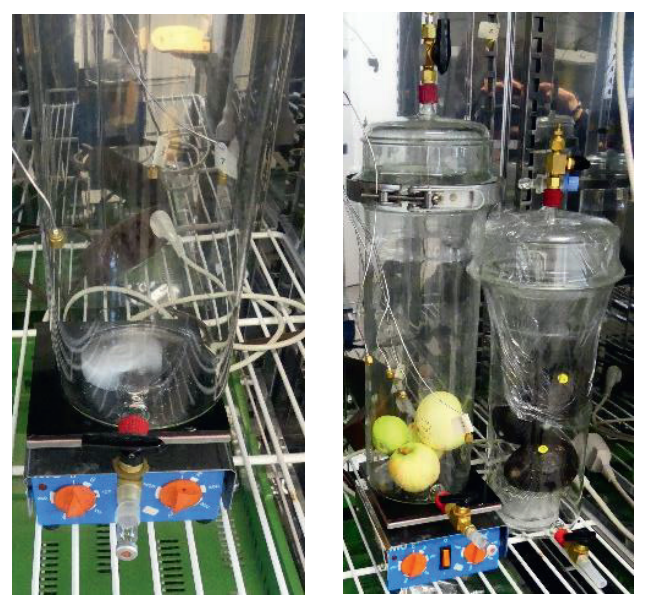

Abb. 1: Gasmesszelle. Links mit Segel am Magnetrührer, Rechts mit Äpfeln und Avocados gefüllt.

Um die reale Anwendung zu überprüfen, wurden drei Äpfel (unbehandelt, Einwaage $322 \mathrm{~g}$ ) in die Gasmesszelle gelegt und deren Gasemission gemessen (Abb. 1). Dazu wurde dreimal bei $32{ }^{\circ} \mathrm{C}$ eine Probe entnommen. Eine weitere Probennahme erfolgte $7 \mathrm{~h}$ nach Beginn der Messung bei ebenfalls $32{ }^{\circ} \mathrm{C}$. Der Ethenpeak wurde wie bei der Störgasmessung mittels der Basisdaten aus dem van't Hoff Diagramm bestimmt. Das dargestellte Beispiel ist aus der Messungen nach $7 \mathrm{~h}$. Außerdem wurden Avocados (3 Stück, Einwaage $837 \mathrm{~g}$ ) in die Gasmesszelle eingelagert und deren Gasemission ebenfalls gemessen (Abb. 1). Dazu wurde an Tag 6, 8 und 11 je dreimal bei $32^{\circ} \mathrm{C}$ eine Probe entnommen. Der Ethenpeak wurde wie bei den vorangegangenen Messungen mittels der Basisdaten aus dem van't Hoff Diagramm bestimmt. Die dargestellten Beispiele sind für die Messungen aus Tag 8. 


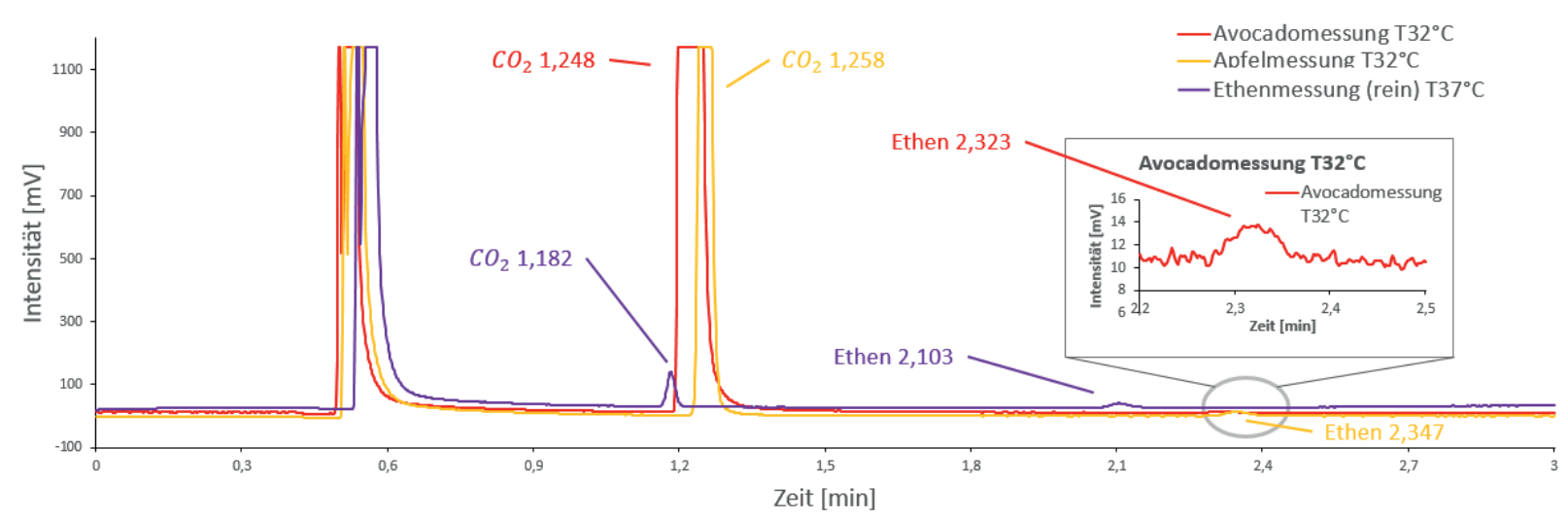

Abb. 2: Chromatogramme aus Apfelmessung (gelb), Avocadomessung (rot) und Ethenmessung (rein, lila)

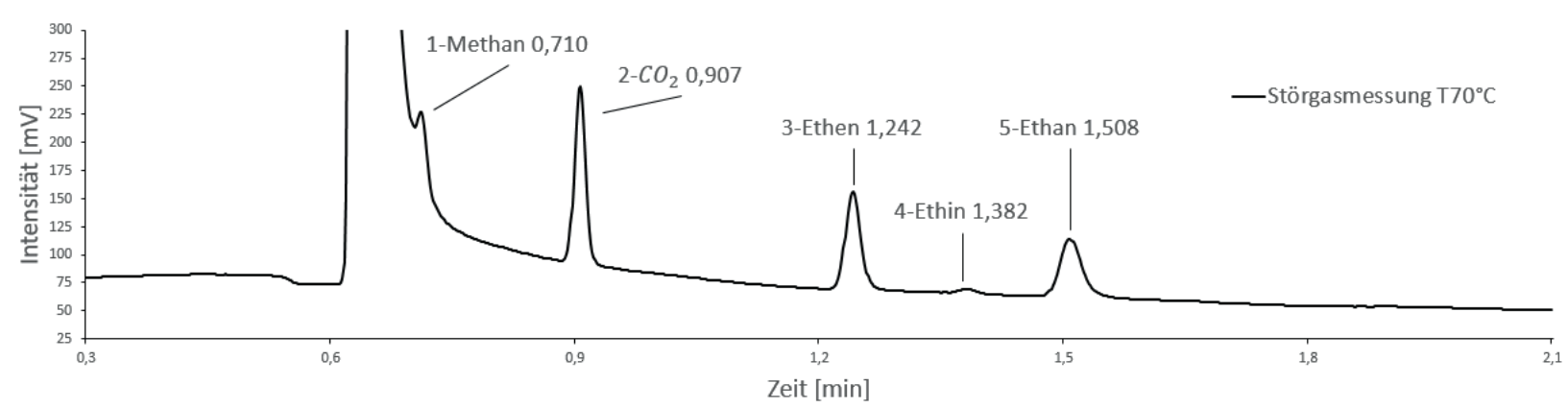

Abb. 3: Chromatogramm der Störgasmessung

\section{Ergebnisse}

Die ersten Messungen des reinen Ethens und des $\mathrm{CO}_{2}$ unter verschiedenen Temperaturen liefern die Basisdaten, aus denen sich das spezifische van't Hoff Diagramm ergibt (siehe Abb. 4). Die daraus ermittelten Enthalpien und Entropien für $\mathrm{CO}_{2}$ und Ethen auf der entsprechenden GS-Q Säule wurden anschließend zur Ermittlung der Prognoseretentionszeiten via (6) verwendet.

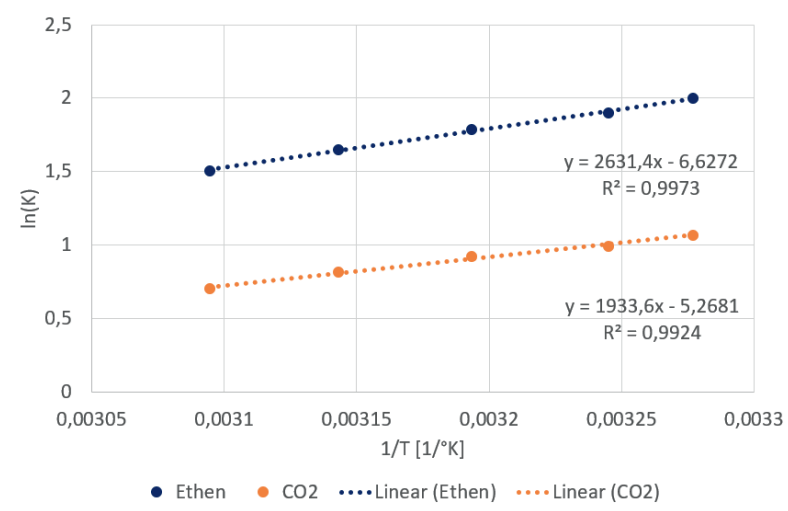

Abb. 4: van't Hoff Diagramm für Ethen (blau) und $\mathrm{CO}_{2}$ (gelb) auf der GS-Q Säule
Dabei wurden für die Enthalpie Werte von 16.075,95 $\left[\frac{\mathrm{J}}{\mathrm{mol}}\right]$ $\left(\Delta H_{\mathrm{CO} 2}^{0}\right)$ und von $21.877,46\left[\frac{\mathrm{J}}{\mathrm{mol}}\right]\left(\Delta H_{\text {Ethylen }}^{0}\right)$ ermittelt. Für die Entropie ergaben sich die Werte von $-43,80\left[\frac{\mathrm{J}}{\mathrm{mol}}\right]\left(\Delta S_{\mathrm{CO} 2}^{0}\right)$ und -55,10 $\left[\frac{\mathrm{J}}{\mathrm{mol}}\right]\left(\Delta S_{\text {Ethylen }}^{0}\right)$. Die Messung mit Störgasen bei verschiedenen Säulentemperaturen zeigen, dass eine Identifikation des Testgaspeaks bei Anwesenheit weiterer unbekannter Peaks ebenfalls möglich ist.

Tab. 2: Gemessene und prognostizierte Retentionszeiten Ethen

\begin{tabular}{|c|c|c|c|}
\hline & $\begin{array}{c}\text { RT CO} \\
\text { gemessen } \\
\text { [min] }\end{array}$ & $\begin{array}{c}\text { RT Ethen } \\
\text { gemessen } \\
{[\text { min] }}\end{array}$ & $\begin{array}{c}\text { RT Ethen } \\
\text { prognostiziert } \\
\text { [min] }\end{array}$ \\
\hline $\begin{array}{c}\text { Blank } \\
\text { T 37 }{ }^{\circ} \mathrm{C}\end{array}$ & 1,182 & 2,103 & 2,151 \\
\hline $\begin{array}{c}\text { Störgase } \\
\text { T } 70^{\circ} \mathrm{C}\end{array}$ & 0,907 & 1,242 & 1,264 \\
\hline $\begin{array}{c}\text { Apfel } \\
\text { T 32 }{ }^{\circ} \mathrm{C}\end{array}$ & 1,258 & 2,347 & 2,390 \\
\hline $\begin{array}{c}\text { Avocado } \\
\text { T 32 }{ }^{\circ} \mathrm{C}\end{array}$ & 1,248 & 2,323 & 2,427 \\
\hline
\end{tabular}

Ein Beispiel ist bei einer Messung der Störgase mit einer Säulentemperatur von $70{ }^{\circ} \mathrm{C}$ in Abbildung 3 zu finden. Anschließend wurde eine Messung der Avocados und eine 
Messung der Äpfel bei mehreren Temperaturen durchgeführt. In beiden Fällen ließ sich ein Ethenpeak detektieren. Die Messergebnisse für $32{ }^{\circ} \mathrm{C}$ sind in Abbildung 2 zu finden. Die auf Basis der finalen Gleichung prognostizierten Retentionszeiten und die tatsächlich gemessenen Retentionszeiten sind in Tabelle 2 nebeneinander dargestellt.

\section{Diskussion}

Bei Betrachtung der Ethen-Blankmessung ist zu erkennen, dass der prognostizierte Peak mit RT Ethen 2,151 min. dem tatsächlich gemessenen Peak mit 2,103 min. sehr nahe liegt (RTCO2 lag bei 1,182 min.). Ebenso fällt bei der Störgasmessung auf, dass die berechnete $\mathrm{RT}_{\text {Ethen }}$ von 1,264 min. eindeutig auf dem Ethenpeak (bei 1,242 min.) liegt ( $\mathrm{RT}_{\mathrm{CO}_{2}}$ lag bei 0,907 min.). Durch die Abhängigkeit der beiden Retentionszeiten (Testgas und Referenzgas) voneinander kann so eine Prognose über die $\mathrm{RT}_{\text {Ethen }}$ ausgehend von der $\mathrm{RTCO}_{2}$ auf dieser Säule durchgeführt werden.

Die Messung der pflanzlichen Proben (Äpfel in Raumluft) zeigt ebenfalls eine klare Übereinstimmung der gemesse-

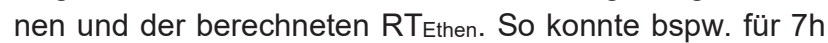
bei Raumtemperatur gelagerte Äpfel ein RTEthen von 2,390 min. bei einer Säulentemperatur von $32{ }^{\circ} \mathrm{C}$ berechnet werden, während der gemessene Wert bei 2,347 min lag (RT $\mathrm{CO}_{2}$ lag bei 1,258 min.). Für die Avocados wurde ein Wert von 2,427 min. prognostiziert, der tatsächlich gemessene Wert war mit 2,323 min. dem beinahe übereinstimmend ( $R T_{\mathrm{CO}_{2}}$ lag hier bei 1,248 min.).

Die auftretenden, kleinen Abweichungen, die sich meist in der zweiten und dritten Nachkommastelle ergeben, könnten durch Messungenauigkeiten des GCs, wie eine schwankende Raumtemperatur in der Probe, oder auf eine sich verändernde Luftfeuchtigkeit zurückzuführen sein. Die in den Rechnungen verwendete Totzeit zur Unterscheidung zwischen Brutto- und Nettoretentionszeit ist vermutlich eine weitere mögliche Ursache der Abweichungen in den prognostizierten Retentionszeiten. Da die experimentelle Totzeit etwas variiert, wurde eine mittlere Totzeit zur Bestimmung der Nettoretentionszeiten verwendet. Die dadurch entstehenden Abweichungen beeinflussen den Fehler in der Prognose zusätzlich. Daher sollte in einem zukünftigen Anwendungsfall eine vorher genaue Bestimmung der Totzeit durch eine kontinuierlich exakte Probennahme sichergestellt werden.

$\mathrm{Da}$ insgesamt jedoch zu jeder Prognose eindeutig ein Peak zugeordnet werden konnte, ist die hier aufgezeigt Methode zur Berechnung der Retentionsindices in Abhängigkeit der Retentionsindizes weiterer Gase gut umsetzbar. Die Vorhersage der RT Ethen erfolgte mit guter Genauigkeit, unabhängig der tatsächlichen Säulentemperatur (hier exemplarisch bei zweimal $32^{\circ} \mathrm{C}, 37^{\circ} \mathrm{C}$ und $70{ }^{\circ} \mathrm{C}$ aufgezeigt), unabhängig der Anwesenheit von Störgasen, oder unabhängig des Ethenursprungs (pflanzlich oder chemisch). Diese einfache und präzise Temperaturkompensation ermöglicht damit die Realisierung kostengünstiger und effektiver GC Systeme.

\section{Danksagung}

Die Arbeiten wurden vom Bayerischen Staatsministerium für Wirtschaft, Landesentwicklung und Energie gefördert im Rahmen des Projekts „Campus der Sinne“.

\section{Interessenskonflikte}

Die Autoren bestätigen hiermit, das keinerlei mögliche Interessenskonflikte bei der Erstellung dieser Arbeit vorlagen.

\section{Literatur}

[1] V. Paul, R. Pandey, Role of internal atmosphere on fruit ripening and storability-a review, Journal of food science and technology (2014), 51, 7, $1223-1250$.

[2] De Biasio, M., Leitner, R., Krall, C., Krivec, M., Wilk, A., Mizaikoff, B., Waldner, R., Starmans, F. \& Maier, D., Ethylene gas sensing using non-dispersive infrared spectroscopy, IEEE Sensors J. (2016), $1-3$.

[3] A. Sklorz, Dow, AB Alamin, D. Mrugala, R. Jedermann, W. Benecke, W. Lang, In-situ Ethylen-Konzentrationsmesssysteme für die Qualitätsüberwachung in der Fruchtlogistik, DFMRS Fachtagung (2008), 50.

[4] F. Lucklum, S. Janssen, W. Lang, M. J. Vellekoop, Miniature 3D Gas Chromatography Columns with Integrated Fluidic Connectors Using High-resolution Stereolithography Fabrication, Procedia Engineering (2015), 120, $703-706$.

[5] W. Lang, R. Jedermann, D. Mrugala, A. Jabbari, B. Krieg-Brückner, K. Schill, The "Intelligent Container"-A Cognitive Sensor Network for Transport Management, IEEE Sensors J. (2011), 11, 3, $688-698$.

[6] S. Janssen, K. Schmitt, M. Blanke, M. L. Bauersfeld, J. Wöllenstein, W. Lang, Ethylene detection in fruit supply chains, Philosophical transactions. Series $A$, Mathematical, physical, and engineering sciences (2014), 372, 2017.

[7] A. Sklorz, S. Janssen, W. Lang, Application of a miniaturised packed gas chromatography column and a $\mathrm{SnO} 2$ gas detector for analysis of low molecular weight hydrocarbons with focus on ethylene detection, Sensors and Actuators B: Chemical (2013), 180, $43-49$.

[8] S. Janssen, T. Tessmann, W. Lang, High sensitive and selective ethylene measurement by using a large-capacity-on-chip preconcentrator device, Sensors and Actuators B: Chemical (2014), 197, $405-413$.

[9] S. Janben, T. Tebmann, M. Nieben, A. Sklorz, W. Lang, Large-capacity-on-chip preconcentrator device for selective ethylene measurement below 400 PPBV, In 2013 Transducers \& Eurosensors XXVII: The 17th International Conference on Solid-State Sensors, Actuators and Microsystems (TRANSDUCERS \& EUROSENSORS XXVII), 2771 - 2774. 
[10] N. A. Zaidi, M. W. Tahir, M. J. Vellekoop, W. Lang, A Gas Chromatographic System for the Detection of Ethylene Gas Using Ambient Air as a Carrier Gas, Sensors (Basel, Switzerland) (2017), 17, 10.

[11] R. Jedermann, T. Poetsch, W. Lang, Smart Sensors for the Intelligent Container, In Smart SysTech 2014; European Conference on Smart Objects,

Systems and Technologies (pp. 1-2). IEEE, 1-2.

[12] A. Eberhardt, M.-L. Bauersfeld, K. Schmitt, J. Wöllenstein, Filterrotationsspektrometer für den Nachweis von Ethen im ppb-Bereich, tm - Technisches Messen (2020), 87, 3, 164 - 176. 